\title{
Graphene photonics for optical communications
}

\section{Marco Romagnoli}

Marco Romagnoli, "Graphene photonics for optical communications," Proc. SPIE 10945, Broadband Access Communication Technologies XIII, 1094502 (1 February 2019); doi: 10.1117/12.2509232

SPIE. Event: SPIE OPTO, 2019, San Francisco, California, United States 


\title{
Graphene photonics for optical communications
}

\author{
Marco Romagnoli \\ Photonic Networks and Technologies Lab - CNIT, Via G. Moruzzi 1, 56124 Pisa (Italy)
}

\begin{abstract}
Graphene is a suitable material for optoelectronic applications that reveals several advantages and complementarities compared with other technologies. Graphene is a gapless material that absorbs radiation from visible to far-infrared and beyond including the terahertz range. The absorption can be modified by changing the material doping, i.e. Fermi level energy, by applying an external electric field. The change in absorption can be very fast and, for this reason, graphene can be used to realize optical modulators. Moreover, the absorption change occurs along with refractive index change. In particular, conditions, i.e. for Fermi level above the Pauli blocking, light is not absorbed anymore and only phase change occurs. Phase change has been demonstrated to be good for fast Mach Zehnder interferometer based modulation. The broadband absorption of Graphene is also exploited to realize efficient photodetectors. Generation of hot electrons upon light absorption in graphene is the cause of photo-thermal effect (PTE) that leads to photo voltage generation. PTE is an ultrafast process that is used for fast photodetection. In this work, the vision for graphene-based integrated photonics is presented. We review state-of-the-art graphene-based modulators and detectors and outline a roadmap matching the technology readiness requirements with the datacom and telecom market demands. We show that graphene-based integrated photonics could enable ultra-high spatial density, low power consumption for board and intra- data centre connectivity, access networks, metropolitan, core, regional and long-haul optical communications.
\end{abstract}

Keywords: Graphene, integrated photonics, optical communications

\section{INTRODUCTION}

Photonics is a key enabling technology that is continuously growing in terms of markets and applications. Notwithstanding the number of applications, photonics production is not yet comparable to microelectronics in terms of volumes even though most of our communications and many other applications rely on photonics [1]. In optical communications, Telecom and even more Datacom, photonics plays a major role; in fact, all the large bandwidth and connection capacity needs to be optical. The main utilization of photonics is in the transport, metro, access network and in data center interconnections. These applications require volumes not exceeding million devices per year at most. However, in the next years this volume size will change because of a transformation of the connection requirement due to the emerging market of IoT (Internet of Things) [2]. The very large increase of connected devices will require a large and pervasive photonic communication infrastructure and ultra large bandwidth in all layer of the networks [3]. In the $5 \mathrm{G}$ era, that is under development, the supposed increase of bandwidth will be a factor of 1000 with respect to present 4G bandwidth [4]. This will require large bandwidth photonic interconnections starting from wireless antennas down to base stations, to radio access network and so on. In data centres, the interconnections are in between racks whereas in the near future, the optical interconnections will be extended to intra- board interconnection that will increase the volume of photonic devices [5]. In other words, the number of photonic interconnection will grow to tens of million devices per year.

The photonic devices, e.g. modulators and detectors, need to meet strict requirements in terms of size, efficiency and power consumption. Graphene photonics is a new and promising technology, still under development [6]. Graphene photonics provides all the basic components that are required for any communication system, i.e. switches, modulators and photodetectors. Graphene is a gapless material that exhibits both electro- absorption and electro- refraction which can be exploited for light modulation and photodetection [7]. Graphene photonics is compatible with complementary metal oxide semiconductor (CMOS) processing, and enable post-processing fabrication of active devices, e.g. modulators and detectors. Graphene photonics provides significant advantages with respect to standard Silicon Photonics. Practically, the use of Graphene on optical waveguides means a post- processing shift in manufacturing from front- end to back- end-ofline. Moreover, graphene technology does not necessarily require expensive SOI wafers, or implantation for junctions, and Ge growth for detectors. These features imply a simpler technology at reduced costs.

Here we report on recent progresses of Graphene photonics, providing a review of the latest results about modulators and detectors. Section 2 is dedicated to Graphene modulators, while section 3 is dedicated to Graphene photodetectors. 


\section{GRAPHENE PHOTONICS MODULATORS}

Electro-optic modulator is one of the key devices of photonic integrated circuits. Broadband operation at high modulation speed combined with small footprint, low power consumption and minimum insertion loss represent the main challenge for future optical interconnect [8]. Graphene based optical modulators offer several advantages with respect to silicon based modulators: broadband electro-absorption, efficient electro-refractive effect, high speed and athermal optoelectronic properties. Graphene, a zero bandgap material, is a broadband absorber with $2.3 \%$ absorption at normal incidence [9]. However, Graphene can be integrated on top of an optical waveguide to enhance the interaction of the propagating guided mode and improving significantly the light absorption. Moreover, the complex dielectric constant of Graphene, i.e. refractive index and absorption, can be properly changed by varying the electrical carrier concentration through electrical gating, allowing for the realization of broadband electro-absorption modulator (EAM) or phase modulators [10]. The first EAM based on a single layer of graphene on top of a doped Si waveguide was demonstrated in 2011 [11]. The Graphene monolayer was grown by chemical vapour deposition (CVD) and transferred on a $7 \mathrm{~nm}$ thin film of Alumina $\left(\mathrm{Al}_{2} \mathrm{O}_{3}\right)$ covering a doped $\mathrm{Si}$ ridge waveguide. The resulting Graphene- $\mathrm{Al}_{2} \mathrm{O}_{3}-\mathrm{Si}$ capacitor is the active device, where an external voltage applied to the capacitor allows for the gating of the graphene Fermi level energy. This work experimentally showed for the first time the great advantage of Graphene EAM's with respect to the Franz Keldish (FK) and quantum confined stark effect (QCSE) modulators. Typical Si photonics FK and QCSE EAM based on SiGe or III-V on Si are limited by the bandgap of the material requiring different devices for different operating wavelength with a typical bandwidth of $30 \mathrm{~nm}$. On the contrary, Graphene allowed broadband operation over hundreds of nanometers from the telecom $\mathrm{O}$ band beyond the $\mathrm{C}$ band towards the mid and far infrared.

The industry's first Graphene on SOI EAM capable of $10 \mathrm{~Gb} / \mathrm{s}$ non-return-to-zero NRZ modulation was demonstrated in 2016 [12]. In this device, the authors used a single layer of CVD grown graphene separated from the doped Si ridge waveguide by $5 \mathrm{~nm}$ thin $\mathrm{SiO}_{2}$ layer in order to create $\mathrm{Graphene}_{-} \mathrm{SiO}_{2}-\mathrm{Si}$ capacitor stack. The authors demonstrated open eye diagrams at $10 \mathrm{~Gb} / \mathrm{s}$ with extinction ratio as high as $2.5 \mathrm{~dB}$, a peak-to-peak driving voltage of $2.5 \mathrm{~V}$, small foot-print $\left(50 \mathrm{x} 10 \mu \mathrm{m}^{2}\right)$ and energy consumption as low as $350 \mathrm{fJ} / \mathrm{bit}$. However, this kind of modulators has a strong limitation due to the use of implantation of dopants in the Si waveguide in order to achieve high speed of operations. An improved design is obtained by implementing the gating capacitor by means of two layer of graphene separated by a thin dielectric film. In this way, the optical waveguide can be a simple passive waveguide of any material. The double layer graphene modulator was demonstrated for the first time in 2012 [13]. Recently, two layers of graphene separated by a $65 \mathrm{~nm}$ thin film of $\mathrm{Al}_{2} \mathrm{O}_{3}$ were used on a $\mathrm{Si}_{3} \mathrm{~N}_{4}$ microring resonator to realize a $22 \mathrm{~Gb} / \mathrm{s}$ electro-optic modulator based on resonator loss modulation at critical coupling [14]. The device is based on the Zeno effect induced by tuning the optical absorption of Graphene on top of a Si microring thus changing the critical coupling condition of the resonance. The authors demonstrated $15 \mathrm{~dB}$ static ER with a record modulation bandwidth of $30 \mathrm{GHz}$.

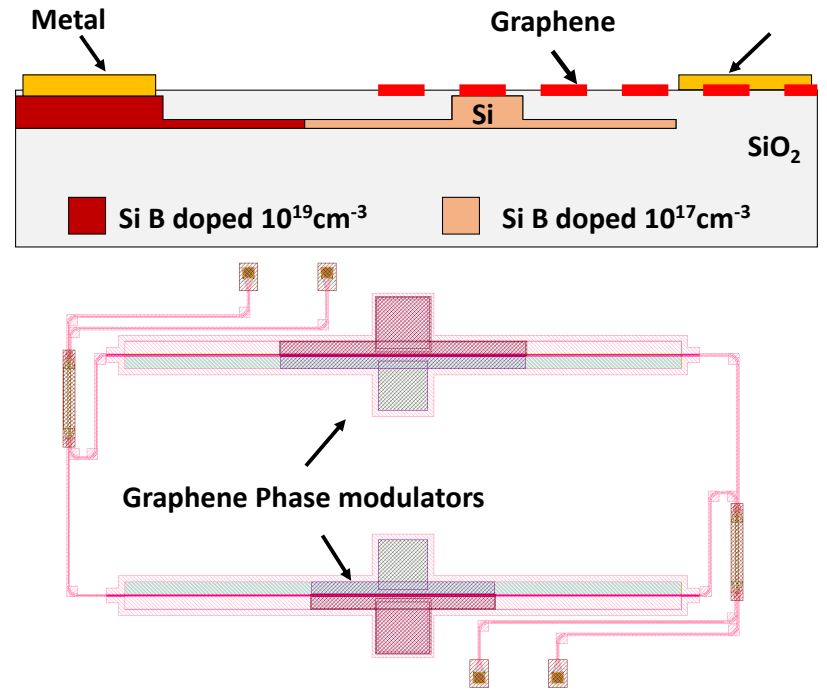

(a)

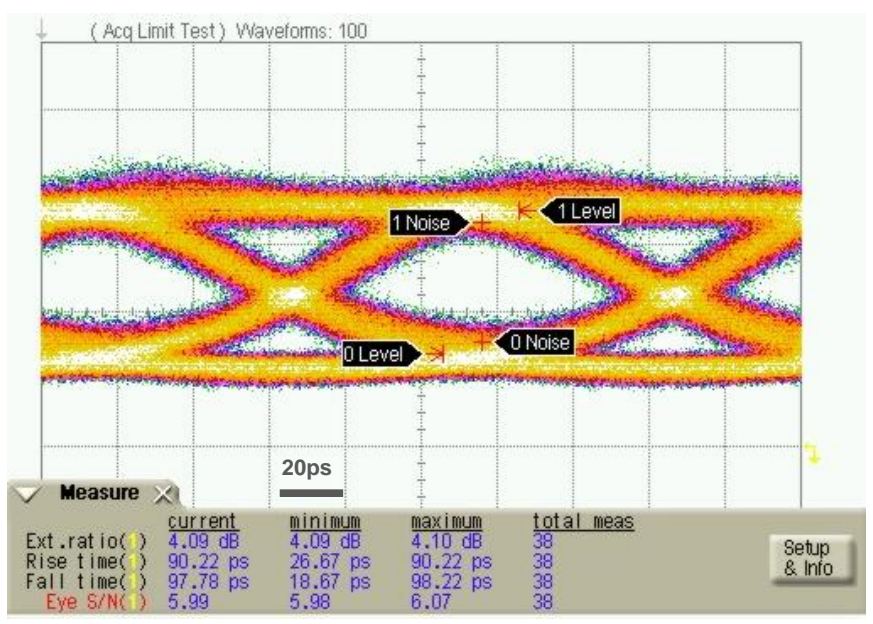

(b)

Figure 1. (a) Cross-section of the device and MZI layout. (b) NRZ eye diagram at 10Gb/s 
Graphene can be used also for the realization of efficient phase modulators. These are of great interest in order to exploit complex modulation formats as for example QPSK and QAM, or in order to realize MZI modulators with low insertion loss and large extinction ratios. We demonstrated that phase modulation in single layer graphene on Si EAM introduces a chirp that is beneficial in compensating the chromatic dispersion of single mode optical fibres. This effect was exploited to demonstrate optical transmission of a $10 \mathrm{~Gb} / \mathrm{s} \mathrm{NRZ} \mathrm{data} \mathrm{stream} \mathrm{up} \mathrm{to} 100 \mathrm{~km}$ single mode optical fibre link [15]. Phase modulation has been also demonstrated in in single layer graphene on Si modulators integrated in MZI up to 10Gb/s [16]. Figure 1 shows the cross section of the modulator and the demonstrated results. We also investigated the double layer structure on both $\mathrm{Si}$ and $\mathrm{Si}_{3} \mathrm{~N}_{4}$ waveguides to demonstrate efficient and fast modulators. Figure 2 shows the cross section of the proposed device.
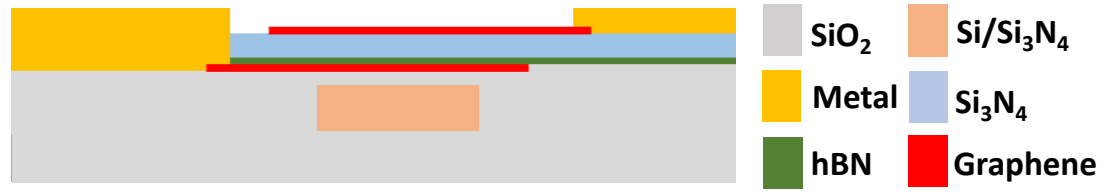

Figure 2. Cross-section of the double layer graphene modulator integrated on $\mathrm{Si}$ or $\mathrm{Si}_{3} \mathrm{~N}_{4}$ waveguides

Graphene has been grown with a predetermined seeded array approach [17] and the resulting single crystal graphene had a defined size that was changed from 100 to $200 \mu \mathrm{m}$ depending on the application. The crystals were then transferred with a semi-dry approach to the waveguides previously planarized with a residual cladding thickness of $10 \mathrm{~nm}$ on to the top of the core. The flatness of the planarized surface was determined with AFM measurement to be 0.6.nm. The single crystal transferred on this surface was analysed with a Raman probe and the carrier mobility resulted to be $7000 \mathrm{~cm}^{2} \mathrm{~V}^{-1} \mathrm{~s}^{-1}$. A commercial $\mathrm{hBN}$ single layer was transferred on the graphene crystal. The role of $\mathrm{hBN}$ is only for protection during the step of $10 \mathrm{~nm}$ thin layer PECVD $\mathrm{Si}_{3} \mathrm{~N}_{4}$ deposition that was used as spacer in the capacitor. The second single crystal of graphene was transferred on top of $\mathrm{Si}_{3} \mathrm{~N}_{4}$; the full stack was then patterned for metallization. Two Ni/Au contacts were then evaporated on the pad areas. For the SOI waveguide the same stack and contact scheme were used. A number of graphene EA and MZI modulators have been realized on $\mathrm{Si}_{3} \mathrm{~N}_{4}$ waveguides. The cross section of the $\mathrm{Si}_{3} \mathrm{~N}_{4}$ waveguide was $250 \mathrm{~nm} \times 1500 \mathrm{~nm}$. Figure 3 shows optical microscope pictures of the fabricated devices and the obtained NRZ eye diagrams. The modulators were electrically driven at $3.5 \mathrm{~V}_{\mathrm{pp}}$ with a $2^{31}-1$ pseudo random binary sequence (PRBS) from the pattern generator at $10 \mathrm{Gbs}, 20 \mathrm{~Gb} / \mathrm{s}$ and $25 \mathrm{~Gb} / \mathrm{s}$.

(a)

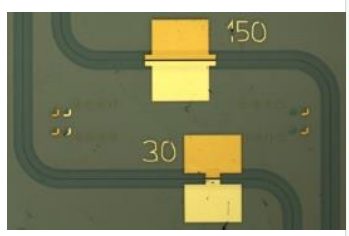

(b)

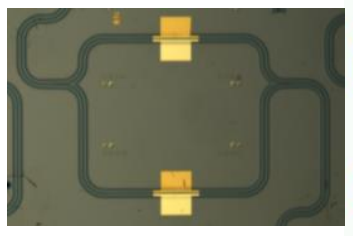

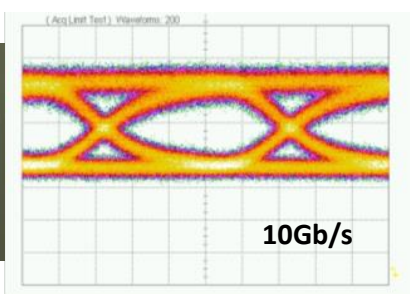

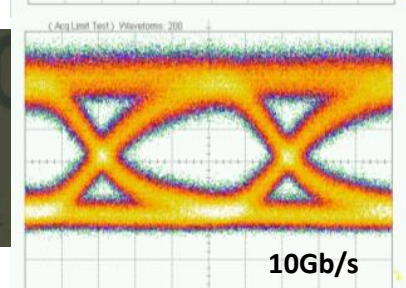

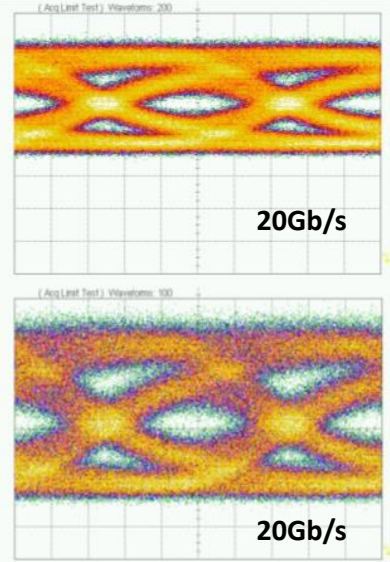

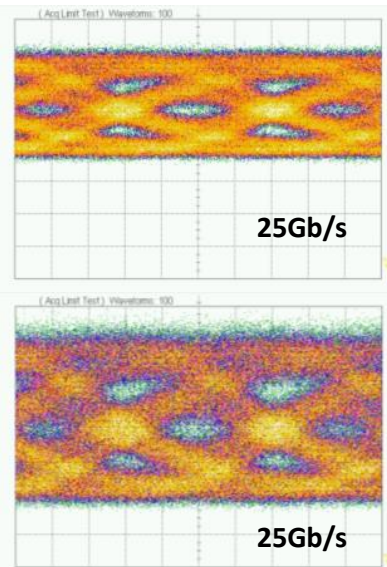

Figure 3. (a) Optical microscope image of a fabricated double layer graphene EAM on $\mathrm{Si}_{3} \mathrm{~N}_{4}$ waveguide and measured NRZ eye diagrams at $10 \mathrm{~Gb} / \mathrm{s}, 20 \mathrm{~Gb} / \mathrm{s}$ and $25 \mathrm{~Gb} / \mathrm{s}$. (b) Optical microscope image of a fabricated double layer graphene MZI on $\mathrm{Si}_{3} \mathrm{~N}_{4}$ waveguide and measured NRZ eye diagrams at $10 \mathrm{~Gb} / \mathrm{s}, 20 \mathrm{~Gb} / \mathrm{s}$ and $25 \mathrm{~Gb} / \mathrm{s}$.

We also demonstrated double layer EAM modulator integrated on 220nmx480nm SOI nanowires. In this case, the contacts on graphene are closer because of the smaller cross section of the SOI nanowire compared to the $\mathrm{Si}_{3} \mathrm{~N}_{4}$ waveguide. The effective $-3 \mathrm{~dB}$ electro-optical bandwidth of the fabricated double layer EAM on SOI is $\sim 30 \mathrm{GHz}$. The modulator was 
electrically driven at $3.5 \mathrm{~V}_{\mathrm{pp}}$ with a $2^{31}-1$ pseudo random binary sequence from the pattern generator at 10,25 and $50 \mathrm{~Gb} / \mathrm{s}$. Results are shown in Fig. 4

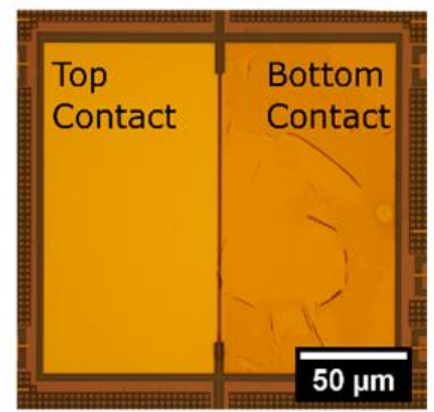

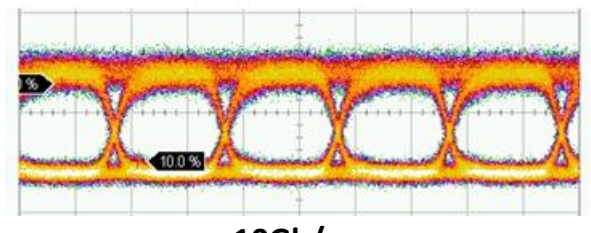

$10 \mathrm{~Gb} / \mathrm{s}$

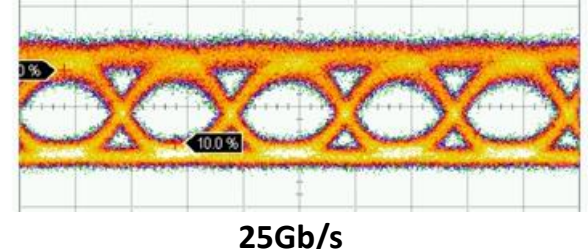

$25 \mathrm{~Gb} / \mathrm{s}$

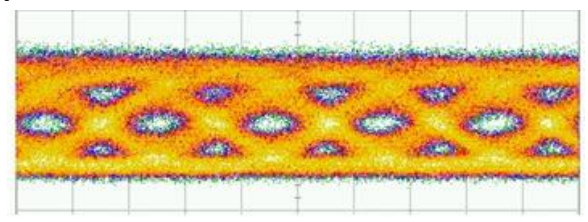

$50 \mathrm{~Gb} / \mathrm{s}$

Figure 4. Optical microscope image of a fabricated double layer graphene EAM on SOI waveguide and measured NRZ eye diagrams at $10 \mathrm{~Gb} / \mathrm{s}, 25 \mathrm{~Gb} / \mathrm{s}$ and $50 \mathrm{~Gb} / \mathrm{s}$.

\section{GRAPHENE PHOTONICS DETECTORS}

Graphene photonics photodetectors should be integrated in a waveguide and optimized in performance as photodetectors relevant for Si photonics. Photodetectors in Si photonics are miniaturized and simple to fabricate photodetectors fulfilling the demands of scalability, footprint, and cost. The graphene photodetectors responsivity $\mathrm{R}_{\mathrm{ph}}$ should be comparable to that of Ge devices in Si photonics, that is $\sim 0.85-1.15 \mathrm{~A} / \mathrm{W}$ at $1,550 \mathrm{~nm}[18]$.

Graphene provides significant advantages for photodetection due to its unique properties [19]: wavelength independent absorption, fast photovoltage generation, fast photo- switching rates, high internal quantum efficiency $>80 \%$. Graphene photodetectors may be based on different detection mechanisms including: photovoltaic (PV) effect [20], photo-thermoelectric (PTE) effect [21] or bolometric effect [22]. Among these, the PTE effect is very promising for the realization of photodetectors with both high-speed (up to $100 \mathrm{GHz}$ ) and efficiency that may exceed $100 \%$ because of hot carrier multiplication [23]. The PTE effect arises in the presence of photogenerated hot carriers generated on a few tens of femtosecond timescale. The photogenerated hot electrons and holes are thermally decoupled from the crystal lattice because of slower electron-lattice relaxation time, in the picosecond time scale [21]. The temperature profile of the hot carriers generated through the optical absorption may be converted in a local photovoltage via the Seebeck effect occurring when the Seebeck coefficient is changed along the graphene layer. This is the case of double-gated devices where the graphene Seebeck coefficient is changed by applying different voltages to different regions of the graphene layer. The photovoltage generated through the PTE effect exhibits significant advantages, e.g. it can be amplified by a voltage amplifier rather than a transimpedance amplifier that allows the realization of photoreceivers at lower cost and with lower power consumption.

The integration of graphene on Si photonic for the realization of photodetectors have been reported. These devices are typically based on a single layer of graphene evanescently coupled to Si waveguides provided with two metal contacts. The guided mode enables longer interaction between graphene and the optical waveguide compared with normal-incidence illumination, allowing for larger optical absorption and responsivity. A graphene photodetector based on the bolometric effect consisting of a CVD grown single layer of graphene on a Si waveguide operating at $1550 \mathrm{~nm}$ has been demonstrated with a $-3 \mathrm{~dB}$ bandwidth of $41 \mathrm{GHz}$ operating at $50 \mathrm{~Gb} / \mathrm{s}$ [24]. A bolometric waveguide-integrated graphene photodetector with plasmonic enhancement has been recently demonstrated with $0.5 \mathrm{~A} / \mathrm{W}$ responsivity at telecom wavelengths and photoresponse faster than $110 \mathrm{GHz}$ [25].

A PTE graphene photodetector integrated on a Si waveguide was also demonstrated with a responsivity of $0.36 \mathrm{~A} / \mathrm{W}$ and $40 \mathrm{GHz}$ bandwidth [26]. The device was obtained by asymmetrically placing the drain and source metal contact with respect to the waveguide core and using a gate to maximize the Seebeck coefficient of the graphene. The single layer graphene was encapsulated in hexagonal boron nitride $(\mathrm{hBN})$ to improve the mobility up to $\sim 40,000 \mathrm{~cm}^{2} \mathrm{~V}^{-1} \mathrm{~s}^{-1}$, therefore the Seebeck coefficient. Double gated PTE photodectors have been also demonstrated. A Si slot-waveguide has been used 
as a dual gate for the realization of a graphene PTE photodetector. The device exhibits extrinsic responsivity of 3.5V/W at zero bias and a $-3 \mathrm{~dB}$ bandwidth of $65 \mathrm{GHz}$ [27]. A similar approach was recently used with a photonic crystal waveguide where the photonic structure is used for mode confinement and as a split-gate electrode in proximity of the optical absorption region. A photoresponsivity of $4.7 \mathrm{~V} / \mathrm{W}$ and a (setup-limited) electrical bandwidth of $18 \mathrm{GHz}$ are achieved [28].

We used the split-gate geometry to realize a PTE graphene photodetector on a $\mathrm{Si}_{3} \mathrm{~N}_{4}$ waveguide. The cross-section of the device is shown in fig. 5 .

\section{(a)}
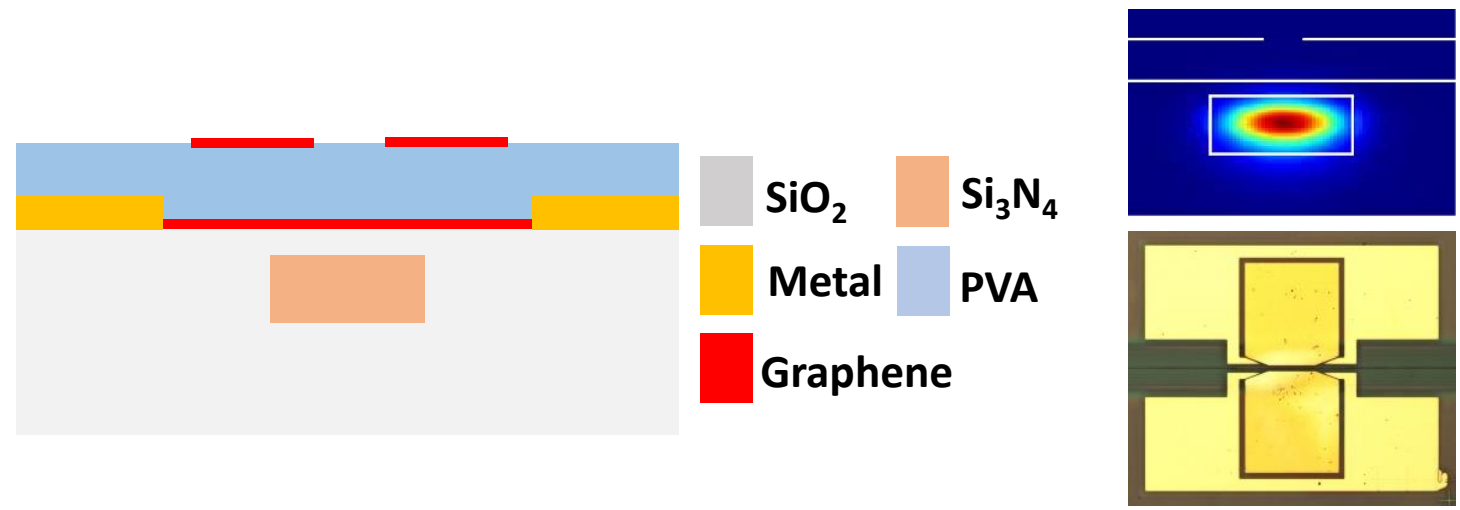

(b)

(c)

Fig. 5: (a) Cross section of the device, (b) Optical mode of the waveguide, c) Optical micrograph of a realized device

The device based on CVD Graphene single crystal transferred by the semi-dry method on top of a single mode transverse electric (TE) $\mathrm{Si}_{3} \mathrm{~N}_{4}$ waveguide with cross-section $1.5 \mu \mathrm{mx} 250 \mathrm{~nm}$. The photodetector is based on a split gate structure in order to create the two regions with different Seebeck coefficients by electrical gating of the bottom graphene with different chemical potentials. The top gates are obtained with a second layer of CVD graphene transferred with the same semi-dry method, and separated from the bottom layer by 120nm thick of polyvinyl alcohol (PVA). The bottom and top graphene are electrically accessible through Nickel/Gold contacts.

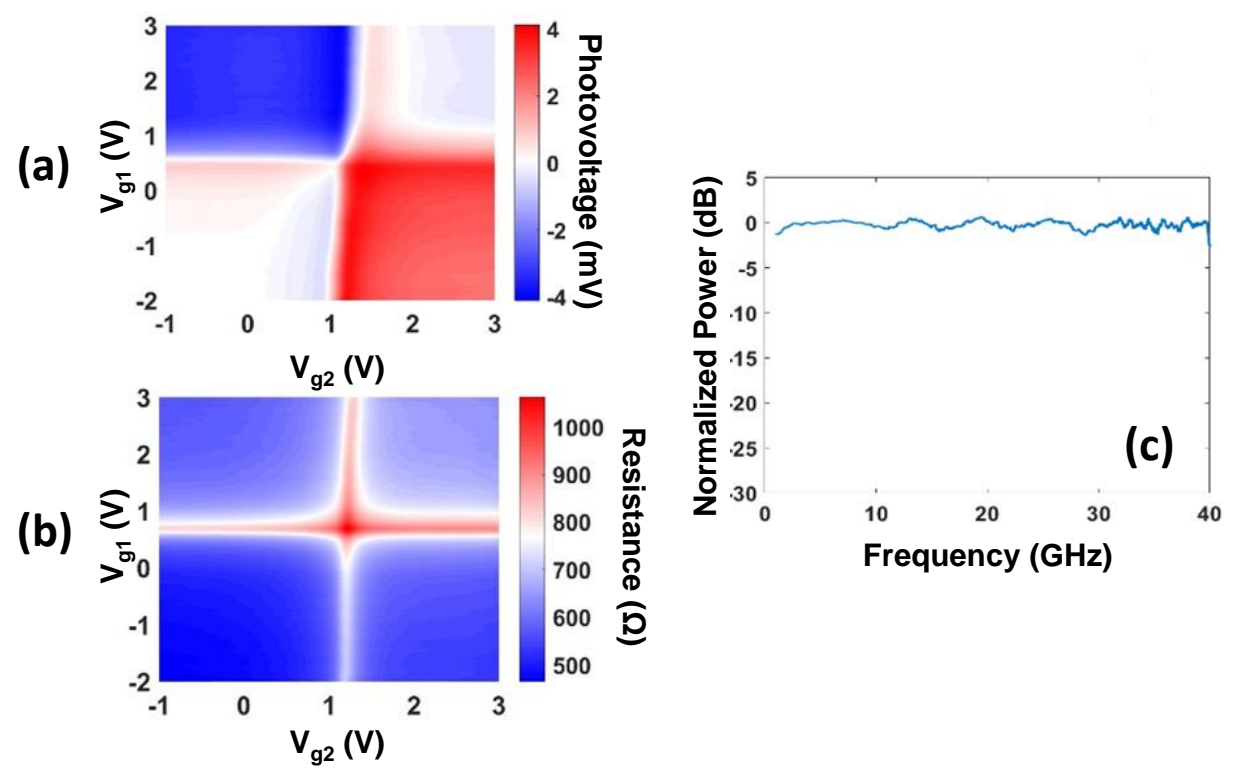

Fig. 6: (a) Photovoltage map as function gate voltages, (b) Resistance map, c) Measured optical bandwidth of the device 
We optimized the cross section of the device by numerical simulations including optical mode analysis at 1550nm (fig. 1(b)). We modelled the PTE effect to describe the case of a detector integrated on a photonic waveguide [29]. The model is defined by the system of equations:

$$
\nabla^{2} T_{e l}+\frac{1}{L_{c}^{2}}\left(T_{e l}-T_{0}\right)=\frac{P_{a b s}(x, z)}{k_{e l} h_{g}} ; \quad \vec{J}=-\sigma \nabla V+\sigma S(\mu(x)) \nabla T_{e l} ; \quad \vec{\nabla} \cdot \vec{J}=0
$$

We characterized the detector at $1550 \mathrm{~nm}$ wavelength in both static and dynamic operations. We measured the DC photovoltage and photoresistance map sweeping the two gate voltages, as well as the electrical bandwidth of the device by using a Mach Zehnder modulator up to $50 \mathrm{GHz}$ and a vector network analyzer (VNA). Figure 6 shows the results.

The measured photovoltage map (fig. 6(a)) exhibits a clear six-folds pattern, which is a clear signature of the PTE effect [29]. The maximum corresponds to a responsivity of $6 \mathrm{~V} / \mathrm{W}$. The resistance map of the device, shown in fig. $6(\mathrm{~b})$, exhibits a maximum, i.e. Dirac point, close to $0 \mathrm{~V}$ that indicates that the transferred graphene is a high quality material. This aspect is very important in order to demonstrate a clear PTE effect.

The electrical bandwidth of the device was obtained using a Lithium Niobate Mach Zehnder modulator with $50 \mathrm{GHz}$ electro-optical bandwidth and a vector network analyzer (VNA). The gate voltages were set to obtain the maximum responsivity. The setup was calibrated using a commercial photodiode with $40 \mathrm{GHz}$ bandwidth. The result shown in fig. 6(c) demonstrates a flat bandwidth up to $40 \mathrm{GHz}$ limited only by the experimental setup. This result is very encouraging because obtained with a wafer-scale process with a CVD material.

\section{CONCLUSIONS}

The results shown in this paper are obtained on a wafer-scale integration process on different photonic platforms employing CVD graphene. We demonstrate that double layer graphene modulators are ready to compare with state-of-the-art photonics integrated devices and paves the way to the establishment of Graphene photonics technology which could enable ultra-high spatial bandwidth density, low power consumption for connectivity within boards or between data centres, access networks and metropolitan, core, regional and long- haul optical communications.

\section{ACKNOWLEDGMENTS}

This work was conceived within the Graphene Flagship project. The authors acknowledge funding from the European Union H2020 GrapheneCore2 Project (contract \#785219). Imec is acknowledged for the fabrication of the single layer devices. Italian Institute of Technology (IIT - Pisa) is acknowledged for the collaboration in the fabrication of the two layer devices.

\section{REFERENCES}

[1] "Luxtera ships one millionth transceiver product," Photonics Spectra, November 2016, http://www.photonics.com/Article.aspx?AID=61153 (November 2016).

[2] Press, G. "Internet of things by the numbers: market estimates and forecasts," Forbes, 22 August 2014, https://www.forbes.com/sites/gilpress/2014/08/22/internet-of-things-by-the-numbers-market-estimates-andforecasts/\#2d4715abb919, (22 August 2014).

[3] M. J. O’Mahony, C. Politi, D. Klonidis, R. Nejabati, and D. Simeonidou, "Future optical networks," J. Lightwave Technol. 24, 4684-4696 (2006).

[4] A. De La Oliva, et al. "Xhaul: toward an integrated fronthaul/backhaul architecture in 5G networks," IEEE Wirel. Commun. 22, 32-40 (2015).

[5] M. Romagnoli et al., "High-bandwidth density optically interconnected terabit/s boards", Proc. SPIE 10560, Metro and Data Center Optical Networks and Short-Reach Links, 105600E (30 January 2018).

[6] M. Romagnoli, et al., "Graphene-based integrated photonics for next-generation datacom and telecom," Nature Rev. Mater. 3, 392-414 (2018).

[7] F. Bonaccorso, Z. Sun, T. Hasan, and A.C. Ferrari, "Graphene photonics and optoelectronics," Nat. Photon. 4, 611-622 (2010).

[8] D.A.B. Miller, "Device requirements for optical interconnects to silicon chips," Proc. IEEE 97, 1166 (2009).

[9] R. R. Nair, et al. "Fine structure constant defines visual transparency of graphene," Science 320, 1308 (2008). 
[10] V. Sorianello, et al. "Complex effective index in Graphene-Silicon waveguides,” Opt. Express 24, 2998429993 (2016).

[11] M. Liu, X. Yin, E. Ulin-Avila, B. Geng, T. Zentgraf, L. Ju, F. Wang, and X. Zhang, “A graphene-based broadband optical modulator, ,Nature 474, 64 (2011)

[12] Y. Hu, et al. "Broadband $10 \mathrm{~Gb} / \mathrm{s}$ operation of graphene electro-absorption modulator on silicon," Laser \& Photonics Reviews 10, 307-316 (2016).

[13] M. Liu, et al. "Double-layer graphene optical modulator," Nano Lett. 12, 1482-1485 (2012).

[14] C. T. Phare, Y. D. Lee, J. Cardenas, and Michal Lipson, http://arxiv.org/abs/1411.2053, (2014)

[15] V. Sorianello, “Chirp management in silicon/graphene electro absorption modulators," Opt. Express 25, 19371-19381 (2017).

[16] V. Sorianello, et al. " Graphene- silicon phase modulators with gigahertz bandwidth,” Nat. Photon. 12, 40-44 (2018).

[17] V. Miseikis, et al., "Deterministic patterned growth of high-mobility large-crystal graphene: a path towards wafer scale integration," 2D Mater. 4, 021004 (2017).

[18]. J. Michel, J. Liu, and L.C. Kimerling, "High-performance Ge- on-Si photodetectors,” Nat. Photon. 4, 527-534 (2010).

[19] F. H. Koppens, et al., Photodetectors based on graphene, other two-dimensional materials and hybrid systems. Nat. Nanotechnol. 9, 780-793 (2014).

[20] T. Mueller, F. Xia, M. Freitag, J. Tsang, and P. Avouris, "Role of contacts in graphene transistors: a scanning photocurrent study," Phys. Rev. B 79, 245430 (2009).

[21] T. J. Echtermeyer, et al., "Photo- thermoelectric and photoelectric contributions to light detection in metalgraphene-metal photodetectors," Nano Lett. 14, 3733-3742 (2014).

[22] M. Freitag, T. Low, F. Xia, and P. Avouris, "Photoconductivity of biased graphene" Nat. Photon. 7, 53-59 (2012)

[23] K. J. Tielrooij, et al. "Photoexcitation cascade and multiple hot- carrier generation in graphene," Nat. Phys. 9, 248-252 (2013).

[24] D. Schall, et al., "50 GBit/s Photodetectors Based on Wafer-Scale Graphene for Integrated Silicon Photonic Communication Systems," ACS Photonics 1, 781-784 (2014)

[25] P. Ma et al., Plasmonically enhanced graphene photodetector featuring $100 \mathrm{Gbit} / \mathrm{s}$ data reception, highresponsivity and compact size, ACS Photonics Just Accepted Manuscript, DOI: 10.1021/acsphotonics.8b01234

[26] R. Shiue, et al., High- responsivity graphene-boron nitride photodetector and autocorrelator in a silicon photonic integrated circuit. Nano Lett. 15, 7288-7293 (2015).

[27] S. Schuler, at al., "Controlled Generation of a p-n Junction in a Waveguide Integrated Graphene Photodetector," Nano Letters 16, 7107-7112 (2016)

[28] S. Schuler, et al., "Graphene Photodetector Integrated on a Photonic Crystal Defect Waveguide," ACS Photonics 5, 4758-4763 (2018)

[29] J. W. Song et al, "Hot carrier transport and photoresponse in graphene", Nano Lett 2011, 11, 4688-4692. 